\title{
The effect of ozone on wet atmospheric corrosion of aluminium of high-voltage lines
}

\author{
Galina Tatarchenko ${ }^{1}$, Nataliia Beloshitskaya ${ }^{1}$, Viktor Sychenko ${ }^{2,}$, and Vitalii Liashuk ${ }^{2}$ \\ ${ }^{1}$ SNU named after Dal \\ ${ }^{2}$ DNURT, Ukraine
}

\begin{abstract}
Annotation. One of the factors influencing traffic safety is the uninterrupted power supply of interlocking devices. As a rule transmission of electric energy is carried out using aluminum wires. In order to assess the reliability of power lines in the work, research was carried out on the influence of ozone on corrosion of aluminum in a humid atmosphere and acidic medium. It was found that the presence of oxygen dissolved in acetic acid leads to anode currents increase, while at crossover ozone, cathode currents increase by an order of magnitude and 2-3 times the anode decreases; the corrosion potential of an $\mathrm{E}_{\text {cor }}$ shifts to the positive side at $0.15 \mathrm{~V}$. The decrease in the density of anode currents in the presence of ozone proceeds due to the growth of the thickness of the aluminum oxide film. According to the Augerspectra, the film is increased by 3-4 times compared with the nonzonized medium, due to which the corrosion resistance of aluminum $K_{\mathrm{m}}$ increases from 0.066 to $0.025 \mathrm{~g} /\left(\mathrm{m}^{2} \cdot \mathrm{h}\right)$. The rate of formation of oxides depends on the concentration of oxidants in the solution, and on their oxidative activity. According to calculations of thermodynamic factors that determine the preferred route of oxidation, the probability of formation of oxides in the presence of ozone is significantly higher. Local destruction of the protective layer occurs mainly in defective places and places of local increase in $\mathrm{pH}$ of the electrode layer. Investigation of the cyclic polarization of a sample of aluminum, pre-cathode activated by a current of 1 $\mathrm{mA} / \mathrm{cm}^{2}$ density, showed the presence of hysteresis, which decreases in the next 2-3 cycles. In this case, the exterior of the aluminum shifts to $(0.10 \div 0.15) \mathrm{V}$ is more positive and the corrosion rate is reduced, which indicates the formation of an oxide film in which irreversible changes occur. The change in the inclination of the polarization curves indicates that the cathodic reaction is delayed and is associated with the change in the structure of the surface layer of the electrode and the diffusion restrictions of ozone recovery.
\end{abstract}

\section{Introduction}

Atmospheric corrosion is the most common type of corrosion of metals that occurs in a humid air environment: approximately $80 \%$ of metal structures, buildings, structures, bridges, cars, etc. exploited under atmospheric conditions. A distinctive feature of atmospheric corrosion is that it flows not in the volume of the electrolyte, but in thin films. In this case, the corrosion process proceeds according to the laws of electrochemical kinetics, but has its own specific features.

The main factors influencing the speed of atmospheric corrosion are:

- humidity of the atmosphere;

- chemical composition of the atmosphere;

- duration of humidifying and drying periods of moist films.

Wet atmospheric corrosion usually begins at relative humidity above $70 \%$. At this moisture, called critical, there is capillary condensation of moisture and water begins to manifest the properties of the electrolyte.
Capillary condensation can stimulate surface roughness, various inequalities, metal contamination with solid particles (dust), etc. The thickness of the moisture films formed on the metal ranges from 0.01 to $1 \mu \mathrm{m}$ and under these conditions a very intense oxygen flow occurs to the surface of the metal, which leads to an acceleration of the corrosion process compared with the volume of the electrolyte.

At high voltage of the electric power line with a high degree of heterogeneity of the electric field near the surface there is a dissociation of the characteristic form: the region covered by the discharge, has a weak purple glow with non-stationary yellow thin channels of single discharges - streamers. In such a "crown case", the electric field strength is sufficient for the ionization and excitation of neutral gas particles. The appearance of corona in the air is accompanied by sound (noise), in the area of the cover is formed ozone, that is, the source of the active oxidizer. Loss of energy on the crown significantly reduces the economic performance and leads to active corrosion processes of all structures. 


\section{Problem statement}

The deterioration of the power transmission lines in many countries is most often due to corrosion of aluminum wires. As noted in [1], one of the main possible reasons for changing the longitudinal resistance of the transmission lines - corrosion of wires under the influence of various climatic factors (including acid rain, humidity, high temperature, solar radiation). According to their calculations for the conditions of Ukraine, the rate of corrosion of aluminum wires should be taken in the range of $1.75-3.5 \mu \mathrm{m} /$ year (for rural and urban areas). In this case, the longitudinal active resistance of phase aluminum wires consisting of wires of a minimum standard diameter $(1.7 \mathrm{~mm})$, during 30 years of operation can (at maximum corrosion rate) increase by $30 \%$, and in 70 years - double $(100 \%)$. For wires with a maximum wire diameter $(4.1 \mathrm{~mm})$ the degree of growth of the longitudinal resistance decreases and at the same rate of corrosion will be: for 30 years of operation $12 \%$; over 70 years $-26 \%$.

From the point of view of ensuring the reliability of power supply and extending the service life of conductors, new alloys with high corrosion resistance are often developed. For example, as suggested by the authors [2] conductors of aluminum alloy with manganese, which have high resistance to sea salt, their corrosion is 1.6-2 times higher than that of ordinary aluminum. This work is relevant because there is not much literature data on corrosive aluminum studies involving the active oxidant [3] in a humid atmosphere, especially with the participation of ozone formed during discharges.

\section{Analysis of recent researches and publications}

The corrosion resistance of aluminum and its alloys is mainly due to the presence of a protective oxide film $\mathrm{Al}_{2} \mathrm{O}_{3} \cdot 3 \mathrm{H}_{2} \mathrm{O}$ or $\mathrm{Al}_{2} \mathrm{O}_{3} \cdot \mathrm{H}_{2} \mathrm{O}$ [4]. Diluted acetic acid at temperatures up to $50{ }^{\circ} \mathrm{C}$ hardly affects aluminum, that is, in $5 \%$ of $\mathrm{AcOH}$ at a temperature of $200{ }^{\circ} \mathrm{C}$, the metal collapses slowly [4]. The presence in the solution of oxygen-containing oxidizing agents $\left(\mathrm{CrO}_{4}^{2-}, \mathrm{VO}^{3-}\right.$, $\mathrm{MnO}^{4-}, \mathrm{MoO}_{4}{ }^{2-}, \mathrm{WO}_{4}{ }^{2-}$ ) changes the nature of the anode curves of aluminum dissolution - even small $(0.01 \mathrm{~N})$ additives $\mathrm{Cr}_{2} \mathrm{O}_{7}{ }^{2-}$ slows down the overall process compared to the background solution [5]. However, with a further increase in the concentration of the oxidizer, the rate of corrosion is the same as in the background solution, and at concentrations greater than $0.5 \mathrm{~N} \mathrm{Cr}_{2} \mathrm{O}_{7}{ }^{2-}$ activation of the anode reaction occurs. In acidic solutions, oxidizing agents increase the $\mathrm{pH}$ of the electrode layer on aluminum and promote the passivation of the electrode, contributing to a positive shift in the potential of the metal. The presence of protective layers on the surface of the metal (oxide, hydroxide, salt films) leads to a strong polarization of the electrode [6]. It should also be noted that the construction of the model of the mechanism of aluminum corrosion in environments containing oxoanions of oxide type, based on only the study of electrochemical characteristics of the metal is impossible. The identity of the polarization characteristics of aluminum, for example, in acidic and alkaline media [6-8], does not indicate the identity of corrosive properties. The electronegativity of the metal and the amphotericity of its oxides are required in electrochemical studies to account for internal selfdissolving currents, whose magnitude is not always a single-valued function of the concentration of the oxidizer, the properties of the products of restoration and stoichiometric ratios of the components involved in electrochemical reactions.

\section{The purpose of the article}

The purpose of the work is to study the influence of ozone on the corrosive behavior of aluminum in the conditions of the acidic environment of a humid atmosphere.

\section{Materials and methods}

The Electrochemical studies were carried out on samples of aluminum in solutions $5 \%$ acetic acid at a temperature of $20{ }^{0} \mathrm{C}$. Polarization curves were made on electrodes in a three-electrode cell with separated space, an auxiliary electrode - platinum. All potentials are given in the $n$. Ozonation of solutions by passing the gas through the working solution, its concentration in the gas phase was $5 \times 10^{-4} \mathrm{~mol} / \mathrm{l}$. To remove the traces of air-oxide films mechanically cleaned metal samples and steel were preexposed to cathode polarization for 3 minutes with a current density of $100 \mathrm{~A} / \mathrm{m}^{2}$. After the cathodic polarization was completed, cathode and anode curves were taken at a $1.44 \mathrm{~V} / \mathrm{h}$ scan speed, and the anode curves captured in this way were used to estimate the dissolution rate. Extrapolation of curves was carried out to determine the teflon area.

The metallographic studies were carried out on 5 samples after 250 hours of their presence in $5 \%$ of $\mathrm{AcOH}$ with and without ozone.

\section{Results and discussion}

The according to the obtained polarization curves of an aluminum electrode (Fig.1, curve 3), when the nitrogen $\mathrm{E}_{\mathrm{cor}}$ is bubbled through the working solution decreases to $-0.3 \mathrm{~V}$, and the density of currents is reduced by an order of magnitude, that is, without the presence of oxygen in the medium of dissolution is carried out mainly at the expense of acetate ions to form a readily soluble aluminum acetate. The presence of oxygen dissolved in $\mathrm{AcOH}$ leads to the growth of anode currents, and when oxygen or ozone is bubbled, cathode currents increase by an order of magnitude, and the anode decreases 2-3 times, $\mathrm{E}_{\mathrm{cor}}$ shifted to the positive side on $0,15 \mathrm{~V}$.

The presence of ozone in an acid solution increases the corrosion resistance of aluminum, compared with non-ozonized medium, the rate of corrosion of $\mathrm{K}_{\mathrm{m}}$ 
decreases from 0.066 to $0.025 \mathrm{~g} /\left(\mathrm{m}^{2} \cdot \mathrm{h}\right)$ Considering that the $\mathrm{pH}$ of $5 \%$ solution of $\mathrm{AcOH}$ is equal to 2.5 the significant contribution to the process of anodic dissolution of aluminum is made by acetate ions, whose concentration in the working solution is $\sim 5 \cdot 10^{-4}$ $\mathrm{g} \cdot$ ion/liter. The decrease in the density of the anode currents occurs due to the transfer of dissolved oxygen and ozone to the metal and the formation of the oxide layer. The value of the Tafelian coefficient $b_{c}$ for the non-zoned medium, is $0.12 \mathrm{~V}$, corresponding to the stage of the discharge of protons on the metal, in the presence of ozone, the coefficient $b_{c}$ almost twice as high.

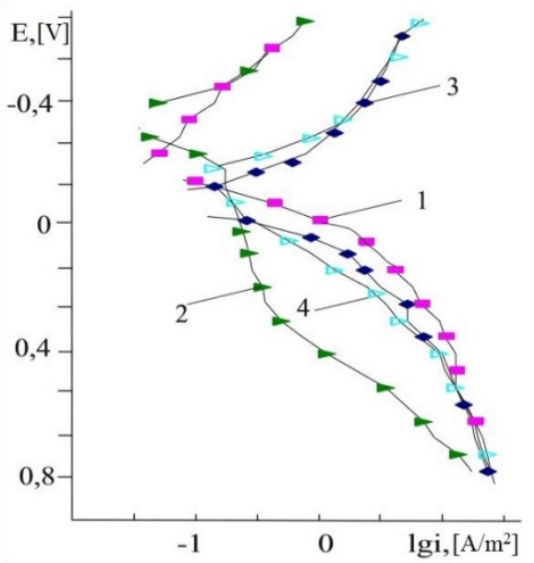

Fig. 1. Polarization curves of aluminum AD0 in $5 \% \mathrm{AcOH}, 20$ ${ }^{\circ} \mathrm{C}$ : 1 - natural aeration, 2 - nitriding, 3 - oxygen, 4 - ozonation of solutions.

However, the calculation of the rate of corrosion on the density of currents obtained from polarization curves shows that with natural aeration $K_{m}$ is $0.025 \mathrm{~g} /\left(\mathrm{m}^{2} \cdot \mathrm{h}\right)$, with bubbling of oxygen through a solution - 0.038 $\mathrm{g} /\left(\mathrm{m}^{2} \cdot\right.$ year $)$, and at bubbling the ozone-oxygen mixture $0,216 \mathrm{~g} /\left(\mathrm{m}^{2} \cdot \mathrm{h}\right)$ Consequently, the electrode eventually changes the oxide films and, accordingly, their conductivity. Such a result is possible in accordance with the proposed hypothesis of the restoration of ozone through its active forms formed in the electrode layer. As a result, it is possible a creation:

$$
\mathrm{Al}+3 \mathrm{OH}^{-} \rightarrow \mathrm{Al}(\mathrm{OH})_{3}+3 e
$$

and further change

$$
2 \mathrm{Al}(\mathrm{OH}) 3+3 \mathrm{H}++3 \mathrm{e}=\mathrm{Al} 2 \mathrm{O} 3 \cdot 3 \mathrm{H} 2 \mathrm{O}
$$

As a result, at the initial moment of time, we obtain anode current growth, due to the strengthening of the cathode reaction of the reduction of ozone, and then its decrease, due to the formation of a thick hydrated oxide film.

As shown earlier, the oxygen and ozone concentrations in the solution can be compared, because the predominant route of oxidation is thermodynamic factors. According to the reference data [5], the most stable form of aluminum oxide when it is formed in water systems is trihydrate $\mathrm{Al}_{2} \mathrm{O}_{3} \cdot 3 \mathrm{H}_{2} \mathrm{O}$, the free energy of formation of which equals $\Delta \mathrm{G}_{298}{ }^{0}=-2295 \mathrm{~kJ} / \mathrm{mol}$. In this regard, the probability of its formation is significantly higher than, for example, monohydrate
$\left(\Delta \mathrm{G}_{298}{ }^{0}=-1427 \mathrm{~kJ} / \mathrm{mol}\right)$. We consider the thermodynamics of two possible ways of formation of aluminum oxides:

ozone $2 \mathrm{Al}+3 \mathrm{H} 2 \mathrm{O}+3 \mathrm{O} 3 \rightarrow \mathrm{Al} 2 \mathrm{O} 3 \cdot 3 \mathrm{H} 2 \mathrm{O}+3 \mathrm{O} 2$

and oxygen $2 \mathrm{Al}+3 \mathrm{H} 2 \mathrm{O}+1.5 \mathrm{O} 2 \rightarrow \mathrm{Al} 2 \mathrm{O} 3 \cdot 3 \mathrm{H} 2 \mathrm{O}$

As calculations have shown, free energy for the reaction

$$
2 \mathrm{Al}+6 \mathrm{H} 2 \mathrm{O} \rightarrow \mathrm{Al} 2 \mathrm{O} 3 \cdot 3 \mathrm{H} 2 \mathrm{O}+3 \mathrm{H} 2,
$$

equals $\Delta \mathrm{G}_{298}{ }^{0}=-336 \mathrm{~kJ} / \mathrm{mol}$ for ozone oxidation of aluminum (3) is $-1044 \mathrm{~kJ} / \mathrm{mol}$, and oxygen (4) - 791.5 $\mathrm{kJ} / \mathrm{mol}$. Given the concentration of ozone and oxygen in the solution, the contribution of ozone in the formation of oxide films and, consequently, in the passivation of aluminum is significant. The rate of formation of oxides depends on the concentration of oxidants in the solution, and on their oxidative activity.

Investigation of the cyclic polarization of the sample Al (Fig. 2), pre-cathode-activated current density 1 $\mathrm{mA} / \mathrm{cm}^{2}$, showed the presence of hysteresis, which decreases in the next 2-3 cycles. In this case, $\mathrm{E}_{\text {cor }}$ of aluminum is shifted by $(0,10 \div 0,15) \mathrm{V}$ is more positive and the rate of corrosion decreases, as evidenced on the formation of an oxide film in which irreversible changes occur, for example, the transition according to the reactions (1-2).

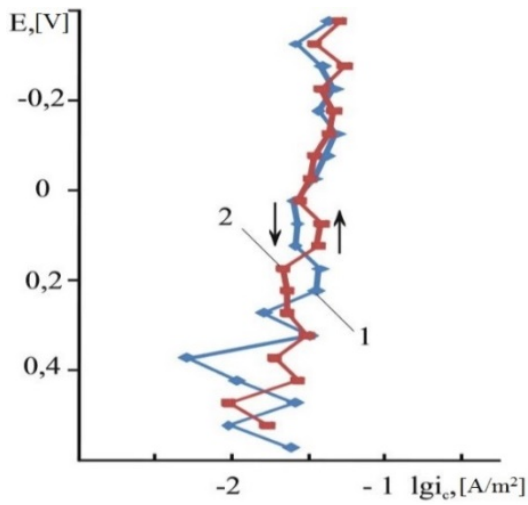

a)

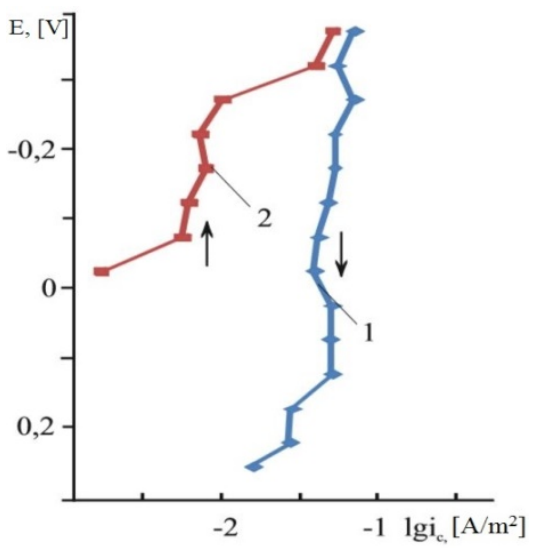

b)

Fig. 2. Cathodic cyclic curves of $\mathrm{Al}$ electrode in ozonated acid solution, $\mathrm{t}=20^{\circ} \mathrm{C}$ : a) the first cycle, b) the sixth cycle, 1 direct polarization, 2 - reverse polarization $(\rightarrow-$ polarization direction indicator) 
In the first cycle, in the cathode polarization of the aluminum electrode in the ozonized acid solution, the curves of the direct and reverse runs practically coincide (Fig. 2). At potentials close to the corrosion potential of aluminum in this medium, unstable values of current density (to a greater extent in direct polarization) are associated with the formation and dissolution of thin oxide layers. Repetitive cyclic polarization practically does not change the density of cathode currents in the forward direction and reduces one and a half or two orders of magnitude with the reverse (Fig.2b).

It is known [9-10] that a corrosion-electrochemical system is capable of generating resonant oscillations, the frequency of which depends on the corrosionelectrochemical properties of the metal. The frequency of oscillations of the corrosion-electrochemical circuit depends on the two-layer capacity of the metal and the polarization resistance. Changes in the sample in accordance with [9] occur primarily due to electrode reactions, which occur in parallel with similar velocities and affect the potential in a double electric layer. On the example of the aluminum sample, it should be noted that the second repetition of cyclic polarization leads to the displacement of the corrosion potential of $\mathrm{E}_{\text {cor }}$ in the direction of positive values. Over time, $\mathrm{E}_{\text {cor }}$ is set to a value of $\approx 0.2 \mathrm{~V}$. In cyclic trials, the anode-cathode transition was fixed at potentials close to $0.55 \mathrm{~V}$, both in direct polarization and in reverse. However, after five to six cycles of $\mathrm{E}_{\text {cor }}$ again set to values close to $0.2 \mathrm{~V}$ (Fig. 2 ). Such changes in the magnitude of the potential for corrosion are possible as a result of the formation of soluble oxide layers on the surface of aluminum in acidic medium.

According to the obtained values of the density of cathode currents in direct and reverse polarization, depending on the number of the cycle, it has been found that in all experiments there is a tendency to increase the currents, the increase of the potential reduces their value and to a greater extent - with direct polarization. The change in the slope of the curves shows that the cathodic reaction is difficult to overcome, which is probably due to the change in the surface layer of the electrode and the diffusion restrictions of oxygen recovery. With anodic polarization of an aluminum electrode, the curves of the direct and reverse move form a negative hysteresis, reaching at a potential of $0.7 \mathrm{~V}$ the difference in the density of currents to one order (Fig. 3). In the case of polarization in the cathodic direction, there is a "delay" in the dissolution of the metal, indicating the formation of a film that protects the surface of the metal well. By the nature of the curves, one can assume the formation of a film with anionic conductivity.

With cyclic polarization, the hysteresis decreases and even tends to form a positive loop and indicates a predisposition to the formation of pittens, which is one of the main factors preventing the use of aluminum as a structural material in the investigated medium. In this case, the angular coefficient of the anode curves is equal to $0.10 \div 0.12$. This testifies to the kinetic complications of the anode process, apparently connected with the metal-electrolyte transformation with the participation of HO-ions. An increase in the size of pittenses, which may be the result of intercrystalline corrosion, is revealed. Changing the anode polarization of an aluminum electrode induces delayed changes in time in the state of its surface, as a result of which the stationary anode activity increases with increasing potential, and decreases with decreasing. Subsequently, the density of the anode currents in the forward direction is reduced to the values of the reverse, therefore, it can be assumed that there are changes in the structure of the oxide film.

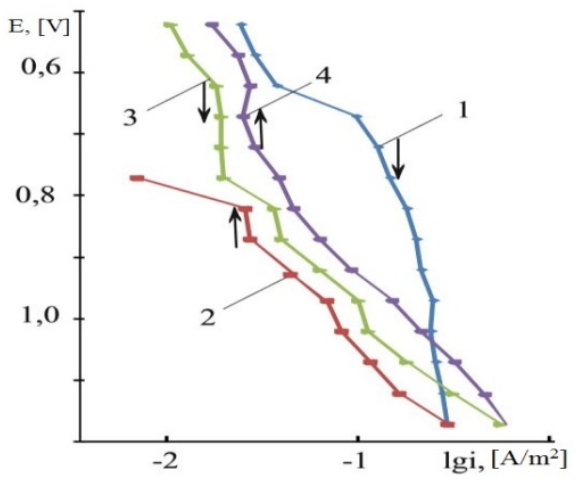

a)

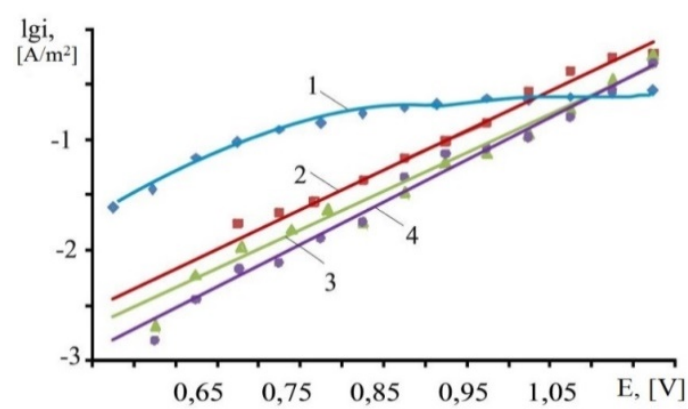

b)

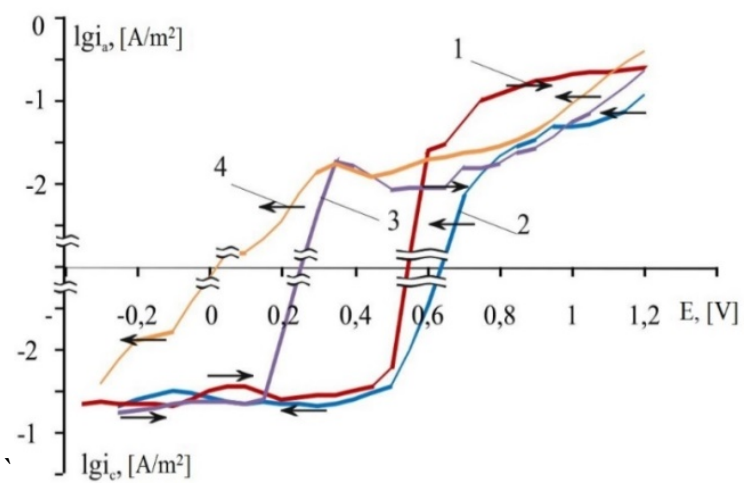

c)

Fig. 3. Cyclic curves of $\mathrm{Al}$ electrode in ozonized acid solution, $\mathrm{t}=20{ }^{\circ} \mathrm{C}$ anode $(\mathrm{a}, \mathrm{b})$ and full $\left.(\mathrm{c}): \mathrm{a}, \mathrm{c}\right) 1,3$ - direct polarization; 2.4 - reverse. 1,2 - the first cycle; 3.4 - the sixth cycle; b) direct polarization; the numbers of the curves correspond to the number of the cycle.

Metallographic studies have shown that the aluminum sills were electroplated, resulting in strips of metal aluminum compounds with refractory impurities. Then, to detect the boundaries of grains, the silt was etched with $0.5 \%$ aqueous solution of hydrofluoric acid (Fig. 4a). It is determined that the boundaries of grains are thin without detecting impurities on them, the grain is large - 1 point on the scale of GOST 5639-82, 
corrosion on grain boundaries was not detected. However, samples after the tests in the ozone medium revealed ulcerous corrosion with a depth of $0.12 \mathrm{~mm}$ (Fig. 4b), the number of ulcers is small and they are chaotic, which can be associated with selective dissolution of microinclusions and heterogeneities in the crystalline structure of the material. The presence of ulcerous corrosion, suggests the existence in the film of pores, where there is an active dissolution of the metal.

Electron microscopic examination of aluminum samples after 24 hours in ozonized acetic acid also revealed a large number of slip lines on the surfaces resulting from preliminary mechanical preparation and proof of these samples. Any obviously pronounced zones or focal points of etching of the surface were absent.

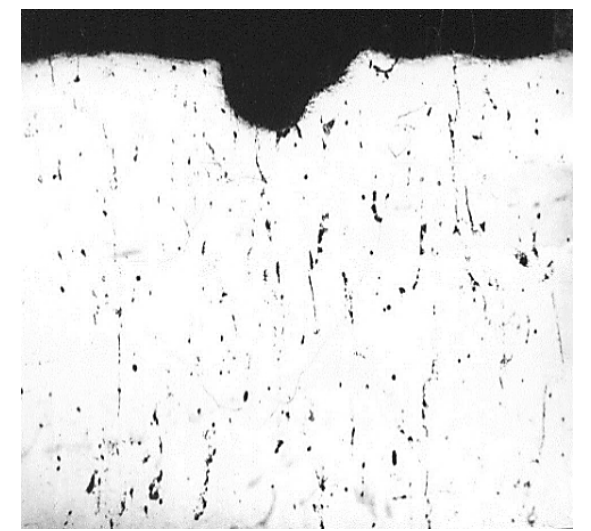

a)

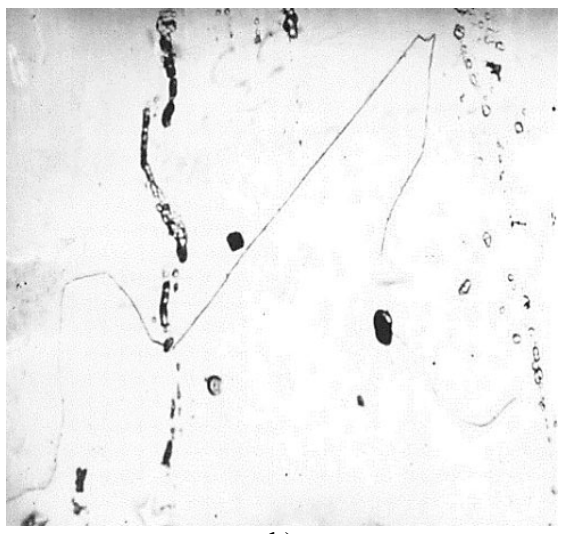

b)

Fig. 4. Microstructure of an aluminum sample after 24 a year stay in ozonated acid solution, $20{ }^{\circ} \mathrm{C}$ : a) $\mathrm{x} 100$; b) $\mathrm{x} 500$.

\section{Conclusions}

Based on the results obtained, we can say that the presence of ozone in an acidic environment:

- reduces the corrosion rate by 2-3 times, it is basically uniform, but in some places characterized by the presence of peptic corrosion;
- substantially changes the thickness of the aluminum oxide film and increases by 3-4 times in comparison with the nonzonized medium;

- leads to the local destruction of the protective layer, especially in defective places and places of local increase in $\mathrm{pH}$ of the electrode layer;

- the rate of formation of oxides depends on the concentration of oxidants in the solution, and on their oxidative activity, according to thermodynamic factors that determine the preferred route of oxidation, the probability of formation of oxides in the presence of ozone is significantly higher.

\section{References}

1. F. P Shkrabets, P. Yu. Krasovsky Influence of aluminum corrosion on electrical parameters of power lines. M. Electromechanics and Automatics: reas.-tech.coll, Iss. 79 - P. 36-39 (2007)

2. M. Watabe, H. Ito, K. Nagano, T. Tsuji, N. Suga and Y. Akasofu. High Corrosion Resistance Conductor for Overhead Transmission Lines Sei Technical Review, No. 84 - P. 47-52 (2017)

3. G. O. Tatarchenko Restoration of ozone and oxygen on metals in the process of corrosion. Bulletin of East-Ukrainian National University named after Volodymyr Dahl №3 (220) - P. 143-149 (2015)

4. A. M. Sukhotin, A. A. Pozdeeva, E. I. Antonovskaya Passivity and corrosion of metals. Proceedings of the GIPH. - L.: Chemistry, Issue.67. - P. 5-12 (1971)

5. V. S. Sinyavsky, V. D. Valkov, V. D. Kalinin. Corrosion and Protection of Aluminum Alloys - M .: Metallurgy. - P. 124-140 (1986)

6. A. M. Sukhotin, Directory of Electrochemistry - L.: Chemistry. - P. 237 (1981)

7. H. Kukhling, Reference book on physics. Trans. from ger. - M .: Mir, - P. 520 (1982)

8. M. S. Smidt, K. K. Baldridge [et. al] General atomic and molecular electronic structure system. J. Comput. Chem - V.4, No. 11 - P.1347-1363 (1993)

9. $\mathrm{Yu}$. N. Mikhailovsky, A. I. Marshakov. Generation of harmonic oscillations by corrosionelectrochemical systems. Protection of metals. V.38. - №1. - P. 5-11 (2002)

10. G. O. Tatarchenko, K. V. Cherkasy Fluctuations of the limiting current in metal dissolution reactions in ozonized sulfuric acid. Fiz.chem material mechanics. - Special issue №7. - Vol.2. - P.911-915 (2008) 